\title{
Influence of chemi-ionization and chemi-recombination processes on hydrogen line shapes in M dwarfs
}

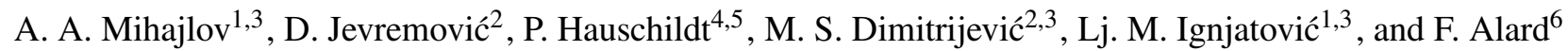 \\ 1 Institute of Physics, PO Box 57, 11001 Belgrade, Serbia \\ e-mail: mihajlov@phy.bg.ac.yu \\ 2 Astronomical Observatory, Volgina 7, 11160 Belgrade 74, Serbia \\ Isaac Newton Institute of Chile, Yugoslavia Branch \\ 4 Depart. of Phys. and Astronomy and Center for Simulat. Physics, University of Georgia, Athens, GA 30602, USA \\ 5 Hamburger Sternwarte, Universitaet Hamburg, Gojenbergsweg 112, 21029 Hamburg, Germany \\ ${ }^{6}$ CRAL/ENS, 46 allée d'Italie, 69634 Lyon Cedex 07, France
}

Received 21 March 2007 / Accepted 20 May 2007

\section{ABSTRACT}

\begin{abstract}
Aims. We study the influence of chemi-ionization in $\mathrm{H}^{*}(n)+\mathrm{H}(1 \mathrm{~s})$ collisions and inverse chemi-recombination processes on the population of higher levels and consequently on profiles of hydrogen lines in the atmospheres of late type (M) stars.

Methods. Modeling, using general stellar atmosphere code PHOENIX to reveal the importance of the inclusion of such processes.

Results. We demonstrate for the first time observationally detectable effects of these chemi-processes on stellar spectra.

Conclusions. It is very important to include chemi-ionization and chemi-recombination processes in modeling of atmospheres of late type stars, especially if one wants to use line profiles for diagnostics of stellar photospheres and lower chromospheres.
\end{abstract}

Key words. atomic processes - molecular processes - stars: atmosphere - line: formation - stars: chromospheres - stars: late-type

\section{Introduction}

We have demonstrated previously (Mihajlov et al. 1992; Mihajlov et al. 1996) the influence of a group of collisional chemi-ionization and chemi-recombination processes on the excited atom populations in hydrogen plasmas with ionization degree less than $10^{-3}$. Here, we have considered namely the ionization processes

$\mathrm{H}^{*}(n)+\mathrm{H} \Longrightarrow \mathrm{e}+\mathrm{H}_{2}^{+}$,

$\mathrm{H}^{*}(n)+\mathrm{H} \Longrightarrow \mathrm{e}+\mathrm{H}+\mathrm{H}^{+}$

and the corresponding inverse recombination processes

$\mathrm{e}+\mathrm{H}_{2}^{+} \Longrightarrow \mathrm{H}^{*}(n)+\mathrm{H}$

$\mathrm{e}+\mathrm{H}+\mathrm{H}^{+} \Longrightarrow \mathrm{H}^{*}(n)+\mathrm{H}$

where $\mathrm{H}=\mathrm{H}(1 \mathrm{~s}), \mathrm{H}^{*}(n)-$ is the hydrogen atom in the excited state with the principal quantum number $n, \mathrm{H}_{2}^{+}-$the molecular ion in the ground electronic state, and e-a free electron. The results obtained were used as a basis to study the processes (1a)-(2b) in the weakly ionized layers of the Solar atmosphere (Mihajlov et al. 1997b) and atmospheres of cool stars (Mihajlov et al. 2003b). The efficiency of these processes was compared with the efficiency of the other relevant ionization and recombination processes, namely, with the electron-atom impact ionization, electron-electron-ion recombination, and electronion photo-recombination processes

$\mathrm{e}+\mathrm{H}^{*}(n) \Longrightarrow \mathrm{e}+\mathrm{e}+\mathrm{H}^{+}$

$\mathrm{e}+\mathrm{e}+\mathrm{H}^{+} \Longrightarrow \mathrm{e}+\mathrm{H}^{*}(n)$
$\mathrm{e}+\mathrm{H}^{+} \Longrightarrow \epsilon_{\lambda}+\mathrm{H}^{*}(n)$

where $\epsilon_{\lambda}$ denotes the energy of the emitted photon. The significant, and often dominant influence of the processes (1a)-(2b) on the populations of excited atom $\mathrm{H}^{*}(n)$ with $n>3$ in the Solar photosphere and lower chromosphere and in considered cool star atmospheres was demonstrated. Previous work has shown the importance of including the above-mentioned processes, given that population numbers can differ by up to $50 \%$ (Mihajlov et al. 2003b).

Investigation of these processes in stellar atmospheres was first reported in Mihajlov et al. (1994). Prior to this, they were mainly investigated for alkali-metal atoms under laboratory conditions. However, they are ab initio included in stellar atmosphere modeling only in Mihajlov et al. (2003). One of the aims of the present paper is to provide, together with that of Mihajlov et al. (2003), enough arguments that these processes are essential for the modeling of stellar photospheres and lower chromospheres for solar-like and cooler stars.

Moreover, in Mihajlov et al. (1997a) chemi-ionization and chemi-recombination processes similar to the processes (1a)-(2b), but in the helium case, were considered, and their influence on the excited atom populations in weaklyionized helium plasma was demonstrated. Subsequently, the importance of such processes on the excited helium atom populations in atmospheres of some DB white dwarfs (Mihajlov et al. 2003a) was shown.

Since the previous results showed that the chemi-ionization and chemi-recombination processes change the population of excited states and the electron density as a function of optical depth in the photospheres and lower chromospheres of solar-like and cooler stars, we consider here the possible influences of the 


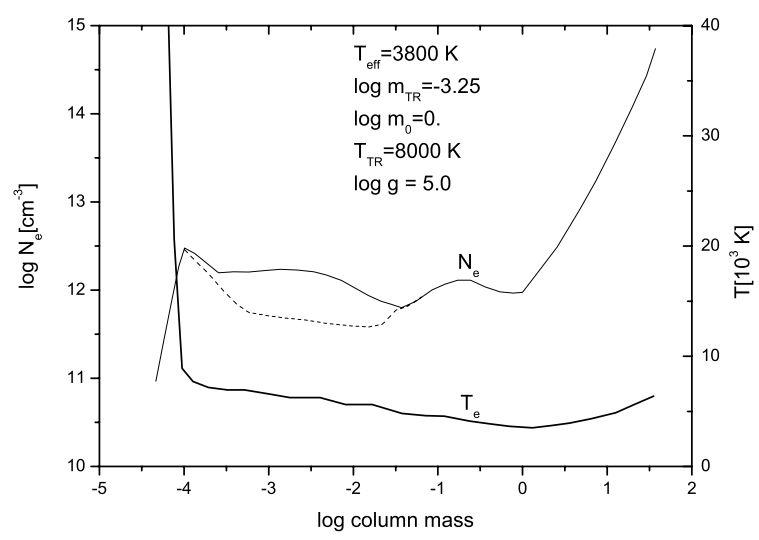

Fig. 1. Structure of model atmosphere - temperature $T_{\mathrm{e}}$ and electron density $N_{\mathrm{e}}$ vs. column mass. Solid line for $N_{\mathrm{e}}$ denotes electron density profile determined with inclusion of chemi-ionization and chemirecombination processes (1a)-(2b), for all $n>1$, and dashed one denotes the electron density profile determined without these processes.

processes (1a)-(2b) on the hydrogen spectral line shapes in nonLTE stellar atmospheres. The stellar atmosphere plasma similar to that examined in Mihajlov et al. (2003b), will be investigated applying similar methodology.

In the case of hydrogen, the method used is correct for $n \geq 5$, when the potential curves of the $\mathrm{H}^{*}(n)+\mathrm{H}$ system are above the potential curves of the $\mathrm{H}^{+}+\mathrm{H}^{-}$system. However the crosspoints of the potential curves of the systems $\mathrm{H}^{*}(n=4)+\mathrm{H}$ and $\mathrm{H}^{+}+\mathrm{H}^{-}$, are so distant that their existence could be ignored so that the method used is applicable for $n \geq 4$. This question was examined in detail in Janev \& Mihajlov (1979) and not considered again in subsequent works. Earlier (Mihajlov et al. 2003), we did not find the rate coefficients for $n=2$ and 3 , since they were not given in direct form in Janev et al. (1987). There, only the data which enable to derive the needed constants are present. So, in the present work we derived them using data from this reference. Also in Mihajlov et al. (2003) our principal aim was to demonstrate that the already-examined processes with $n \geq 4$ are significant for modeling.

Here we introduced in PHOENIX code collisional rates for $n=2$ and 3 obtained on the basis of data from Janev et al. (1987), and thus the theory of processes (1a)-(2b) is completed here.

\section{Results and discussion}

We use the general stellar atmosphere code PHOENIX (Baron \& Hauschildt 1998; Hauschildt et al. 1999; Short et al. 1999) for our modeling. This code has the advantage that apart for solving the atmospheric structure, it also calculates output spectra, so changes in population levels are reflected on line shapes. The procedure similar to that used in Mihajlov et al. (2003b) was applied, but for all $\mathrm{H}^{*}(n)$ atom populations with $n>1$. Rate coefficients are theoretically determined by means of the method developed in Mihajlov et al. (1992, 1996). This method is in principle the same as that developed for the chemiionization processes in the alkali-metal atom collisions, where it is confirmed many times by experiment (see e.g. Ignjatović $\&$ Mihajlov 2005). Basic atmosphere has an effective temperature of $T_{\text {eff }}=3800 \mathrm{~K}$, with chromosphere and transition region appearing at logarithm column mass of 0.04 and -4.0 respectively and temperature at the bottom of transition region fixed at $8000 \mathrm{~K}$. This basic atmosphere is shown in Fig. 1. The

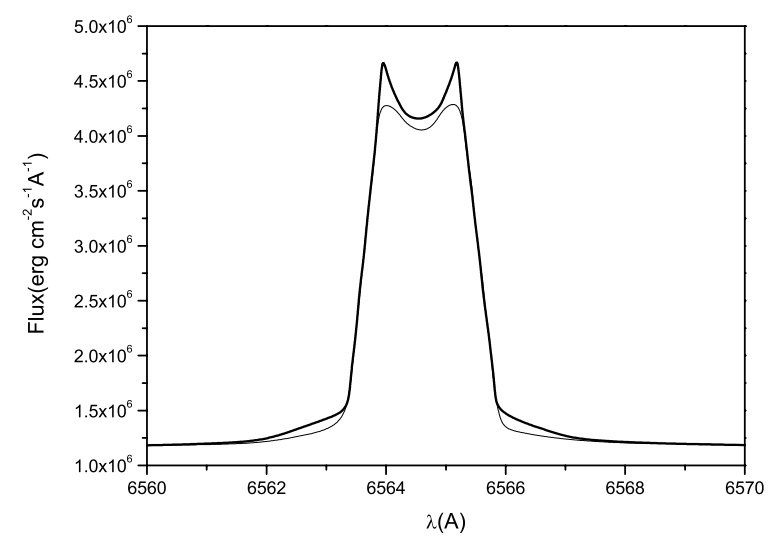

Fig. 2. Line profiles with (full) and without (thin) inclusion of chemiionization and chemi-recombination processes for $\mathrm{H}_{\alpha}$ line from the atmosphere described in text and shown in Fig. 1.

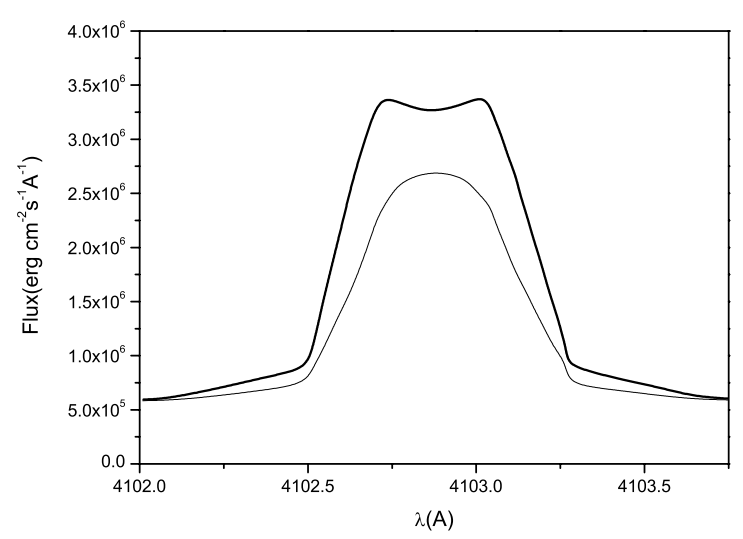

Fig. 3. As in Fig. 2 but for $\mathrm{H}_{\delta}$ line.

solid line for $N_{\mathrm{e}}$ denotes the electron density profile determined with inclusion of chemi-ionization and chemi-recombination processes (1a)-(2b), for all $n>1$, while the dashed line denotes the electron density profile determined without these processes. The hydrostatic equilibrium, the equations of state, and the non-LTE rate equations for $H$ were iterated until changes in populations of levels of atomic hydrogen were less than $1 \%$. We find that inclusion of the new chemi-processes (1a)-(2b) significantly affects the $H$ energy level number densities in M dwarfs, as was found in Mihajlov (2003b) but without $n=2$ and 3 level contribution. Also, the expected change of electron density $N_{\mathrm{e}}$ is confirmed, and found to be particularly important not only when processes (1a)-(2b) with $n>3$ are included, but also the processes with $n=2$ and 3. This is illustrated in Fig. 1. Such changes should also influence spectral line profiles.

In Figs. 2-5 we show the final line profiles of $\mathrm{H}_{\alpha}, \mathrm{H}_{\delta}, \mathrm{H}_{\epsilon} \mathrm{Pa}_{\epsilon}$ with and without inclusion of new processes. Profiles we synthesized with PHOENIX code with Stark broadening calculated using tables from Vidal et al. (1971) for Stark broadening of hydrogen lines (linear Stark effect). The line intensity changes seen in Figs. 2-5 directly demonstrate the influence of the changes of $\mathrm{H}^{*}(n)$ atom populations. Lineshape changes, especially in the wings, show the influence of the electron density change having direct influence on the Stark broadening of hydrogen lines. The particular significance of this result is that it is the first demonstration of the possibility of confirming the influence of chemiionization and chemi-recombination processes (1a)-(2b) by direct observations of stellar spectra. 


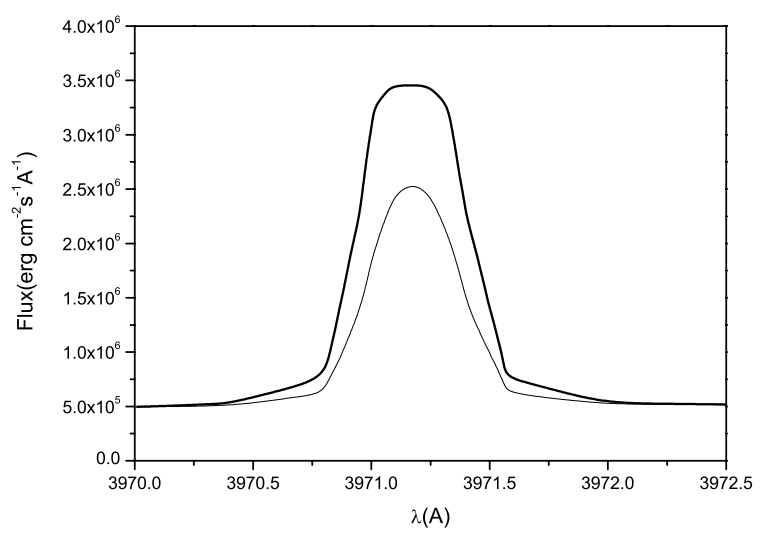

Fig. 4. As in Fig. 2 but for $\mathrm{H}_{\epsilon}$ line.

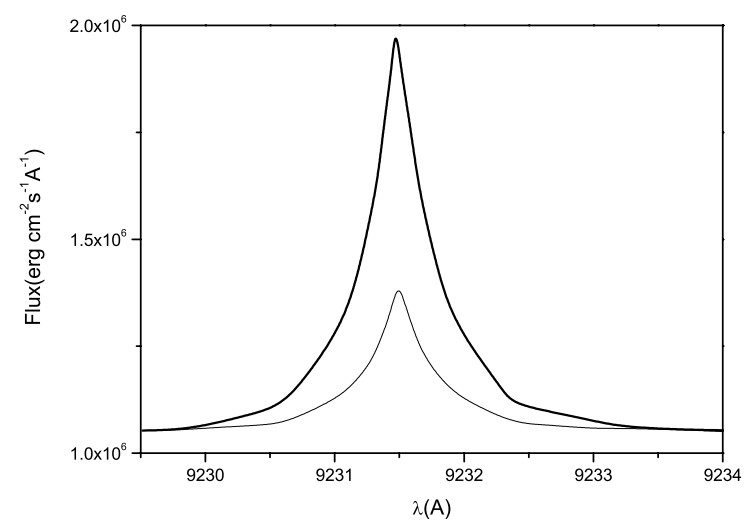

Fig. 5. As in Fig. 2 but for $\mathrm{Pa}_{\epsilon}$ line.

The most important effect of electron density $\left(N_{\mathrm{e}}\right)$ changes must be on the thermalization depth of the line, and hence the brightness in the line core for a given temperature structure. With increased $N_{\mathrm{e}}$, the rate of thermalizing collisions with electrons in the chromosphere will be higher, causing the line center radiative source function to be more closely coupled to the Planck function, hence making the lines righter (i.e. stronger) for a given model structure. This is very important because it systematically affects the diagnostic accuracy of the lines: without inclusion of chemi ionization/recombination processes (1a)-(2b), one would predict emission lines that are too weak from a given model, and, therefore, conclude that the atmospheric pressure is larger than it really is.

Consequently, we can conclude that this is the first demonstration of a significant and observable (measurable) change in line profiles, as a consequence of the influence of the considered processes. Thus it is very important to include chemi-ionization and chemi-recombination processes $(1 a)-(2 b)$ in modeling of atmospheres of late-type stars, especially if one wants to use line profiles for diagnostics of stellar atmospheres. Moreover, it should be taken into account that within the effective temperature range $3000 \mathrm{~K}<T_{\text {eff }}<6000 \mathrm{~K}$, the relative influence of the processes (1a)-(2b) related to other relevant processes (3)-(5) can only increase with the increase of $T_{\mathrm{eff}}$, if the atmospheric composition remains similar to Solar composition. Consequently, for Solar photosphere and lower chromosphere, we should expect the more enhanced influence of the processes (1a)-(2b) than for the case considered here. It should be taken into account however, that within the region where the ionization degree is up to $10^{-3}$, the concurrent processes increase with the increase in temperature, as do electron-atom and electron-ion, as well as radiative processes. So, while $T_{\text {eff }}$ increases, but stays below or close to Solar, on the basis of previous results, (Mihajlov et al. 1997), the increase of the relative influence of chemi-ionization/recombination processes (1a)-(2b) on excited atom populations and electron densities is expected. Generally, according to our knowledge, further improvements to the used method will result in an increase in the influence of chemi-ionization-recombination processes.

Acknowledgements. This work is part of the projects Radiation and transport properties of the non-ideal laboratory and ionospheric plasma (Project number 141033) and Influence of collisional processes on astrophysical plasma lineshapes (Project number 146001) and was supported by the Ministry of Science and Environment Protection of Serbia. We are grateful to Ian Short for very useful suggestions and comments.

\section{References}

Baron, E., \& Hauschildt, P. H. 1998, ApJ, 495, 370

Hauschildt, P. H., Allard, F., \& Baron, E. 1999, ApJ, 512, 377

Ignjatović, Lj. M., \& Mihajlov, A. A. 2005, Phys. Rev. A, 72, 022715

Janev, R. K., \& Mihajlov, A. A. 1979, Phys. Rev. A, 20, 1890

Janev, R. K., Langer, W. D., Evans Jr., K., \& Post Jr., D. E. 1987, Elementary Processes in Hydrogen-Helium Plasmas (Springer-Verlag)

Mihajlov, A. A., Ljepojević, N. N., \& Dimitrijević, M. S. 1992, J. Phys. B, 25, 5121

Mihajlov, A. A., Dimitrijević, M. S., Ignjatović, Lj. M., \& Djurić, Z. 1994, A\&AS, 103, 57

Mihajlov, A. A., Dimitrijević, M. S., \& Djurić, Z. 1996, Phys. Scr., 53, 159

Mihajlov, A. A., Dimitrijević, M. S., Djurić, Z., \& Ljepojević, N. N. 1997a, Phys. Scr., 56, 631

Mihajlov, A. A., Ignjatović, L. M., Vasilijević, M. M., \& Dimitrijević, M. S. 1997b, A\&A, 324, 1206

Mihajlov, A. A., Ignjatović, L. M., Dimitrijević, M. S., \& Djurić, Z. 2003a, ApJS, 147,369

Mihajlov, A. A., Jevremović, D., Hauschildt, P., et al. 2003b, A\&A, 403, 787

Short, C. I., Hauschildt, P. H., \& Baron, E. 1999, ApJ, 525, 375

Vidal, C. R., Cooper, J., \& Smith, E. W. 1971, J. Quant. Spectrosc. Radiative Transfer, 11, 263 